Dr. med. Volker Mielke im Gespräch mit Prof. Dr. med. Dr. h.c. Enno Christophers FRCP

\section{Klinik und Forschung - gehören sie noch zusammen?}

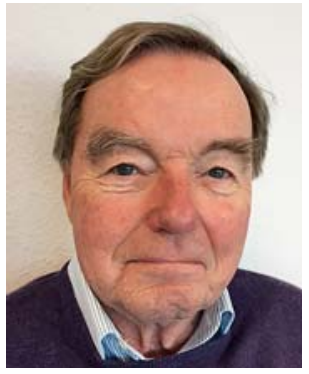

Warum haben Sie die Dermatologie als Fachgebiet gewählt?

Prof. E. Christophers: In der Zeit nach dem Staatsexamen war ich zunächst überzeugt, für die väterliche Allgemeinpraxis in Ostfriesland geschaffen zu sein, von der Dermatologie als Hauptberuf weit entfernt. Das Fach befand sich damals noch im Stadium der reinen Morphologie und Streitereien über die passende Bezeichnung einer Hautveränderung waren Kennzeichen vieler Kongresse. Meine klinischen Semester in Kiel trugen auch nicht dazu bei, von dem Fach begeistert zu sein.

Es waren Göttinger Studienfreunde, die inzwischen in München an der Dermatologischen Klinik der Ludwig-Maximilians-Universität tätig waren und die eine Stelle für mich bereithielten. Dort hatte ich als sog. Medizinalassistent ein halbes Jahr mit Hautkranken zu tun mit dem Ergebnis, dass der damalige Oberarzt (Prof. Röckl) mir ein einjähriges Stipendium in der Dermatologie der University of Pennsylvania in Philadelphia anbot. Ich ergriff diese Chance und blieb zweieinviertel Jahre. Mein Lehrer war Albert Kligman. Er vermochte wie kein anderer für die Haut und ihre Krankheiten zu begeistern. Und es war die Zeit, als die Dermatologie zu neuen Ufern aufbrach: Pathophysiologie, Biochemie und Infektiologie waren faszinierende, neue Felder. In der Ferne winkte schon die heute fast alles beherrschende Immunologie. Es gelang mir in der Folgezeit nicht mehr, davon loszukommen. Somit war es keine Wahl, sondern wohl eher ein Wink des Schicksals.

Welcher Fall ist Ihnen besonders im Gedächtnis geblieben?

Prof. E. Christophers: Im Gedächtnis verhaftete Fälle imponieren zumeist durch groteske Erscheinungsformen oder durch unerwartete Ergebnisse der Diagnostik oder der Therapie. Unvergesslich bleibt mir das Bild einer älteren Dame, die einer strengen, auf alles verzichtenden Sekte angehörte. So auch auf ärztliche Hilfe. Sie befand sich in einem abgetrennten Raum in einem General Hospital in Philadelphia, von weißen Tüchern abgedeckt, und es roch abscheulich. Mir blieb nur ein kurzer Augenblick der klinischen Inspektion. Was ich sah, entsprach anstelle eines Gesichts einem riesigen Ulkus mit einer zuckenden rosigen Zunge. Wahrlich umwerfend aber war das an einem etwa zehn Zentimeter langen Strang hängende Auge. Es war blau und schaute mich an. Ich nahm mich zusammen und ging hinaus. Oft habe ich mich daran erinnert und über ärztliche Aufgaben, auch als Dermatologe, über den Unsinn religiösen Sektierens und die Frage der fehlenden Infektion in diesem Basaliom nachgedacht.

Von wem haben Sie besonders viel gelernt?

Prof. E. Christophers: Von meinem Lehrer Prof. Braun-Falco und von meinen naturwissenschaftlichen Mitarbeitern, insbesondere Jens Schröder.
Was war der beste Rat, den Sie während Ihrer Karriere erhalten haben?

Prof. E. Christophers: Für einen verhalten agierenden, beobachtenden Ostfriesen: „Blow your horn“! (Kligman).

Was ist momentan die wichtigste Entwicklung in der Dermatologie?

Prof. E. Christophers: Ein Thema für ein Symposium! Um kurz zu bleiben: Es sind m.E. drei Bereiche, die wichtige Entwicklungen vermitteln: 1. die Onkologie einschließlich Epidemiologie, Früherkennung, Melanom-Therapie, 2. die Erkennung von Signalwegen bei entzündlichen Erkrankungen und 3. die im Augenblick noch zaghaften Versuche einer wissenschaftlichen Bearbeitung der Kosmetischen Dermatologie.

\section{Wo sehen Sie die Zukunft der Dermatologie?}

Prof. E. Christophers: 1. Zunächst einmal im weiteren wissenschaftlichen Fortschritt - bedingt durch technische Neuentwicklungen werden unsere Kenntnisse in einem, so glaube ich, atemberaubenden Tempo weiterwachsen (wie in der gesamten naturwissenschaftlichen Medizin). 2. Unsere therapeutischen Verfahren werden weiter bereichert, vor allem durch systemische und biologische Therapeutika. Manche Vorgehensweisen sind schon jetzt revolutionär, und das ist noch nicht das Ende. 3. Es droht die Gefahr, dass die universitäre, in den Hautkliniken betriebene Dermatologie durch politische und verwaltungstechnische Zwänge ausgehungert wird. Damit verlieren die Zentren der Weiterbildung und Weiterentwicklung unseres Faches ihre akademische Färbung. Krankenversorgung verlagert sich in noch stärkerem Maße auf außeruniversitäre Zentren. Das wäre vertretbar, würden nicht mit dem Wegfall der universitären Versorgung durch Bettenabbau und poliklinische Einschränkungen sogleich Planstellen reduziert. Anstelle diese Stellen für Forschung und Lehre freizumachen! Auch ein Thema für ein Symposium!

\section{Was raten Sie jungen Kollegen?}

Prof. E. Christophers: Nach dem Abschluss des Studiums einmal in einer Universitätshautklinik anzuklopfen, um sich über eine Promotion oder ein Stipendium (davon gibt es viele) mit einer wissenschaftlichen Fragestellungen zu beschäftigen. Man lernt sich selbst kennen, man findet Gelegenheit in sich hineinzuhorchen, ob man da hineinpasst und wie faszinierend es sein kann, eine Antwort auf eine einfache Frage, die uns die Natur stellt, zu erarbeiten.

Ähnliches gilt für den jungen Kollegen in (und nach Abschluss) der Weiterbildung: Wissenschaft kann unglaublich faszinierend sein, und sie gibt es auch in der klinischen Praxis. Wissenschaft steckt auch in dem besonderen Fall, der ungewöhnlichen Erkrankung.

Was machen Sie nach Feierabend als Erstes?

Prof. E. Christophers: Jetzt ist Feierabend und ich mache mir als Erstes einen Drink. 